\title{
THE SPITZER SOUTH POLE TELESCOPE DEEP FIELD: SURVEY DESIGN AND INFRARED ARRAY CAMERA CATALOGS
}

\author{
M. L. N. Ashby ${ }^{1}$, S. A. Stanford ${ }^{2,3}$, M. Brodwin ${ }^{4}$, A. H. Gonzalez ${ }^{5}$, J. Martinez-Manso ${ }^{5}$, J. G. Bartlett ${ }^{6}$,

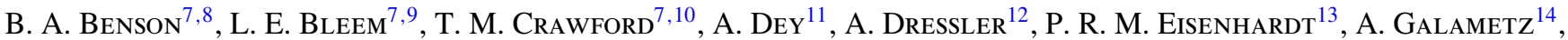

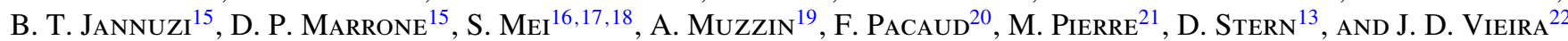 \\ ${ }^{1}$ Harvard-Smithsonian Center for Astrophysics, 60 Garden Street, Cambridge, MA 02138, USA; mashby @ cfa.harvard.edu \\ ${ }^{2}$ Department of Physics, University of California, One Shields Avenue, Davis, CA 95616, USA \\ ${ }^{3}$ Institute of Geophysics and Planetary Physics, Lawrence Livermore National Laboratory, Livermore, CA 94551, USA \\ ${ }^{4}$ Department of Physics and Astronomy, University of Missouri, Kansas City, MO 64110, USA \\ ${ }^{5}$ Department of Astronomy, University of Florida, Gainesville, FL 32611, USA \\ ${ }^{6}$ AstroParticule et Cosmologie, Université Paris Diderot, CNRS/IN2P3, CEA/IRFU, Observatoire de Paris, Sorbonne Paris Cité, \\ 10, rue Alice Domon et Léonie Duquet, F-75205 Paris Cedex 13, France \\ ${ }^{7}$ Kavli Institute for Cosmological Physics, University of Chicago, 5640 South Ellis Avenue, Chicago, IL 60637, USA \\ ${ }^{8}$ Enrico Fermi Institute, University of Chicago, 5640 South Ellis Avenue, Chicago, IL 60637, USA \\ ${ }^{9}$ Department of Physics, University of Chicago, 5640 South Ellis Avenue, Chicago, IL 60637, USA \\ ${ }^{10}$ Department of Astronomy and Astrophysics, University of Chicago, 5640 South Ellis Avenue, Chicago, IL 60637, USA \\ ${ }^{11}$ National Optical Astronomy Observatories, 950 North Cherry Avenue, Tucson, AZ 85719, USA \\ 12 The Observatories of the Carnegie Institution for Science, 813 Santa Barbara Street, Pasadena, CA 91101, USA \\ ${ }^{13}$ Jet Propulsion Laboratory, California Institute of Technology, 4800 Oak Grove Drive, Mail Stop 169-221, Pasadena, CA 91109, USA \\ ${ }^{14}$ INAF-Osservatorio di Roma, Via Frascati 33, I-00040 Monteporzio, Italy \\ ${ }^{15}$ Department of Astronomy and Steward Observatory, University of Arizona, 933 North Cherry Avenue, Tucson, AZ 85719, USA \\ ${ }^{16}$ GEPI, Observatoire de Paris, Section de Meudon, 5 Place J. Janssen, F-92190 Meudon Cedex, France \\ ${ }^{17}$ Université Paris Denis Diderot, F-75205 Paris Cedex 13, France \\ ${ }^{18}$ Infrared Processing and Analysis Center, Pasadena, CA 91125, USA \\ ${ }^{19}$ Leiden Observatory, Leiden University, P.O. Box 9513, 2300-RA Leiden, The Netherlands \\ ${ }^{20}$ Argelander-Institue für Astronomie, Auf dem Hügel 71, D-53121 Bonn, Germany \\ ${ }^{21}$ Service d'Astrophysique, AIM IRFU/DSM/CEA, F-91190 Gif sur Yvette, France \\ ${ }^{22}$ California Institute of Technology, 1200 East California Boulevard, Pasadena, CA 91125, USA \\ Received 2013 July 12; accepted 2013 October 8; published 2013 November 13
}

\begin{abstract}
The Spitzer South Pole Telescope Deep Field (SSDF) is a wide-area survey using Spitzer's Infrared Array Camera (IRAC) to cover $94 \mathrm{deg}^{2}$ of extragalactic sky, making it the largest IRAC survey completed to date outside the Milky Way midplane. The SSDF is centered at $(\alpha, \delta)=(23: 30,-55: 00)$, in a region that combines observations spanning a broad wavelength range from numerous facilities. These include millimeter imaging from the South Pole Telescope, far-infrared observations from Herschel/SPIRE, X-ray observations from the XMM XXL survey, near-infrared observations from the VISTA Hemisphere Survey, and radio-wavelength imaging from the Australia Telescope Compact Array, in a panchromatic project designed to address major outstanding questions surrounding galaxy clusters and the baryon budget. Here we describe the Spitzer/IRAC observations of the SSDF, including the survey design, observations, processing, source extraction, and publicly available data products. In particular, we present two band-merged catalogs, one for each of the two warm IRAC selection bands. They contain roughly 5.5 and 3.7 million distinct sources, the vast majority of which are galaxies, down to the SSDF 5 $\sigma$ sensitivity limits of 19.0 and 18.2 Vega mag (7.0 and $9.4 \mu \mathrm{Jy})$ at 3.6 and $4.5 \mu \mathrm{m}$, respectively.
\end{abstract}

Key words: catalogs - galaxies: clusters: general - infrared: galaxies - surveys

Online-only material: color figures, machine-readable tables

\section{INTRODUCTION}

The large-scale distribution of both baryonic and dark matter and the physical laws that govern their evolution are fundamental to cosmology. Observations of the cosmic microwave background constrain the baryon-to-matter ratio to be $\sim 16 \%$ (Story et al. 2012; Hinshaw et al. 2013; Planck Collaboration et al. 2013). Observations of galaxy clusters, however, have special advantages for understanding the abundance and distribution of matter, because (1) clusters are large enough that they are expected to retain the cosmic fraction of baryons, and (2) clusters are among the few astrophysical objects for which the three dominant forms of matter can be observed, namely: the gas mass $\left(M_{\text {gas }}\right)$, the stellar mass $\left(M_{\text {star }}\right)$, and the dark matter mass $\left(M_{\mathrm{DM}}\right)$. However, observations of the matter distribution in clusters as a function of physical scale and mass have typically been constrained only at low redshift $(z<0.5)$.
At low redshift, the best measurements of the baryon faction to date account for $\sim 80 \%$ of the cosmic value (for a recent review, see Kravtsov et al. 2009). The baryonic mass is dominated by an intracluster gas component (Vikhlinin et al. 2006; Arnaud et al. 2007; Sun et al. 2009), with stars and galaxies typically contributing roughly one-tenth as much mass (Gonzalez et al. 2007; Giodini et al. 2009; Lin et al. 2012). However, the stellar mass fraction has also been found to be a strong function of cluster mass, with the stellar-to-gas fraction decreasing from $\sim 0.25$ to 0.05 from groups $\left(\sim 5 \times 10^{13} M_{\odot}\right)$ to massive clusters $\left(\sim 10^{15} M_{\odot}\right)$. This can be interpreted to mean that star formation is much more efficient in the lower-mass groups compared to rich clusters (e.g., Muzzin et al. 2012).

In contrast with observations, simulations generally predict that the stellar fraction is approximately constant with cluster mass, and over-predict the amount of stars formed by a factor of $\sim 2-5$ in massive clusters (e.g., Kravtsov et al. 2009). More 
recently, simulations have tried to evoke various types of astrophysical feedback (e.g., supernovae and active galactic nuclei) to suppress star formation and match the observed stellar and intracluster medium fraction, with some success (e.g., Planelles et al. 2013; Battaglia et al. 2013).

Some of the fundamental questions about galaxy and structure formation that still await resolution include:

1. Why is the baryon fraction in cluster gas less than the universal average? Are the missing cluster baryons in stars? Do the observations underestimate the total diffuse stellar mass in the form of intracluster light?

2. How do the relationships between cluster gas, dark matter, and stars evolve with redshift? Given that clusters are built hierarchically from mergers of lower-mass groups, in the simplest scenario one might expect these relations to be significantly steeper at higher redshift.

3. What is the radial distribution of the baryonic components as a function of $M_{\mathrm{DM}}$ and redshift?

4. What is the assembly history of baryons in the most massive structures?

Spitzer's Infrared Array Camera (IRAC; Fazio et al. 2004b) has already been used to carry out several relatively widearea extragalactic surveys, e.g., the Spitzer Wide-area Infrared Extragalactic Survey (SWIRE; Lonsdale et al. 2003), the Spitzer Deep, Wide-Field Survey (SDWFS; Ashby et al. 2009), and the Spitzer Extragalactic Representative Volume Survey (SERVS; Mauduit et al. 2012), covering more than $70 \mathrm{deg}^{2}$ altogether. Among many other things, datasets such as these provide an efficient means to identify statistical samples of galaxy clusters down to low masses even at high redshifts (Eisenhardt et al. 2008; Muzzin et al. 2009; Papovich et al. 2010; Stanford et al. 2012). However, the cluster searches carried out to date are not fully characterized in terms of purity, completeness, or mass proxies (via comparison with cluster samples identified at other wavelengths), hindering interpretations that depend upon either cluster mass or ensemble properties.

Here we describe a new wide-area IRAC survey that will make it possible to combine groundbreaking datasets from the South Pole Telescope (SPT) and XMM with coextensive infrared imaging to address the questions posed above. We call this the Spitzer South Pole Telescope Deep Field (SSDF) survey. The SSDF depth and coverage are compared to those of other major IRAC survey projects in Figure 1. The combination of infrared, millimeter, and X-ray data provides a unique opportunity to simultaneously calibrate the IRAC search techniques via comparison with Sunyaev-Zel'dovich (SZ) and X-ray cluster samples, and to robustly determine the cluster selection functions at the depths simultaneously probed by the three techniques. A limitation of the present generation of X-ray and SZ surveys is that they lack the sensitivity at high redshift to identify clusters down to $10^{14} M_{\odot}$ and below, as is possible with IRAC. Reaching these mass scales at higher redshifts is critical for understanding the assembly history of the present-day cluster population, as these low-mass systems are the direct progenitors of typical presentday clusters, like Virgo. The IRAC component is essential for finding the lowest-mass clusters at all redshifts $z>0.5$ and for determining photometric redshifts for complete galaxy samples (Brodwin et al. 2006). It is also vital for determining galaxy luminosities and sizes within clusters (e.g., Brodwin et al. 2010), and for estimating the stellar masses of all clusters found by any selection technique (e.g., Eisenhardt et al. 2008). Because the SSDF offers an opportunity to bring the X-ray and millimeter

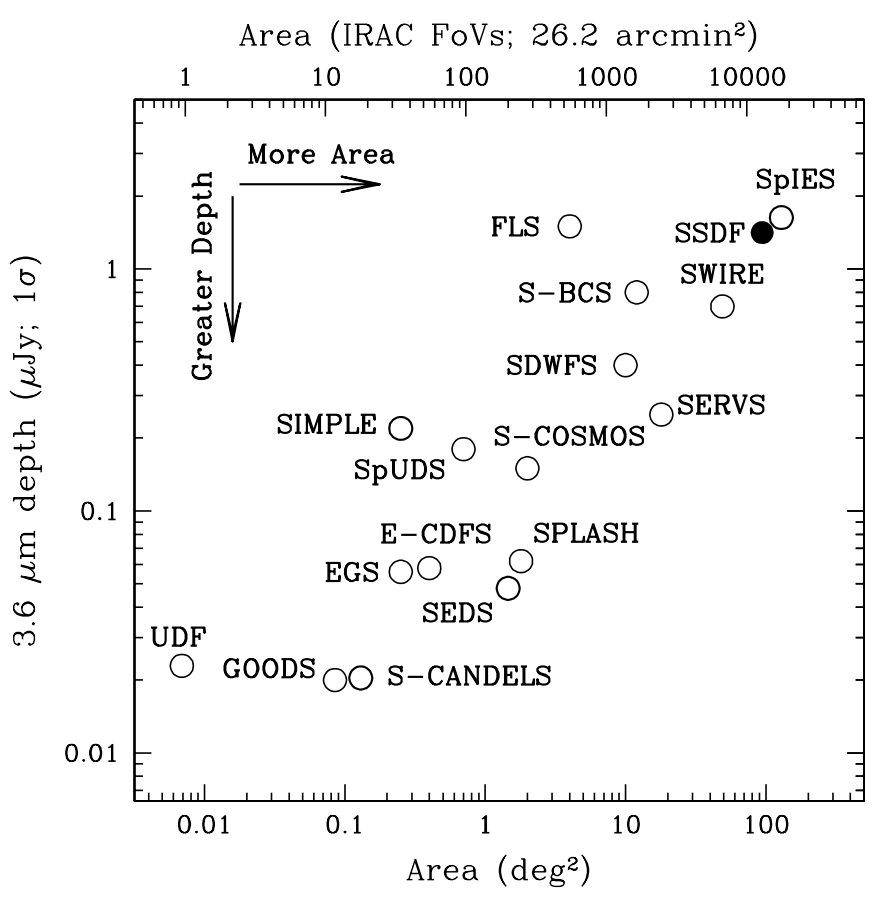

Figure 1. Comparison of SSDF $3.6 \mu \mathrm{m}$ depth and total area (solid circle) to other major Spitzer/IRAC extragalactic surveys. Open symbols indicate $1 \sigma$ point-source sensitivities for GOODS (Great Observatories Origins Deep Survey), EGS (Extended Groth Strip), E-CDFS (Extended Chandra Deep Field South), SpUDS (Spitzer Public Legacy Survey of UKIDSS Ultra-Deep Survey), SCOSMOS (Spitzer Deep Survey of HST COSMOS 2-Degree ACS Field), SERVS (Spitzer Extragalactic Representative Volume Survey), S-BCS (Spitzer-Blanco Cosmology Survey), SWIRE (Spitzer Wide-area Infrared Extragalactic Survey), the FLS (Spitzer First-Look Survey), SDWFS (Spitzer Deep, Wide-Field Survey), SEDS (Spitzer Extended Deep Survey), S-CANDELS (Spitzer-CANDELS), and the Spitzer-IRAC Equatorial Survey (SpIES). All sensitivities shown are based on low-background estimates made with the Spitzer Sensitivity and Performance Estimation Tool (SENS-PET) except for SpIES (which used a high-background estimate), and the SSDF and SEDS, which are measured from actual data.

imaging together with wide-field IRAC data, it is poised to become a uniquely valuable resource for galaxy cluster research.

In this contribution, we describe the Spitzer/IRAC survey: the field, the observations, the reduction, and in particular the resulting catalogs that will serve as the basis for future work aimed at addressing the unresolved questions surrounding the distribution of baryons in the universe. This paper is organized as follows: Section 2 describes the SSDF field and previous observations relevant to galaxy cluster science. Section 3 discusses the details of the SSDF observing strategy and data reduction, and Section 4 describes the source identification, photometry, and validation. Section 5 describes the SSDF catalogs. In Section 6 we present preliminary results, including wide-field infrared number counts and the infrared color distribution of IRAC-detected galaxies. We summarize in Section 7. Unless otherwise stated, all magnitudes are given in Vega-relative terms. Users can convert to the AB scale by adding 2.792 and 3.265 mag to the cataloged 3.6 and $4.5 \mu \mathrm{m}$ magnitudes, respectively.

\section{THE SPITZER SOUTH POLE TELESCOPE DEEP FIELD}

We carried out our survey in a field that benefits from an abundance of supporting data from X-ray to radio wavelengths, and which has extremely low levels of Galactic dust emission, being among the cleanest $1 \%$ of contiguous $100 \mathrm{deg}^{2}$ regions on the sky as measured in the $100 \mu \mathrm{m}$ IRAS map (Finkbeiner et al. 1999). The SSDF is centered at $(\alpha, \delta)=(23: 30,-55: 00)$. The 


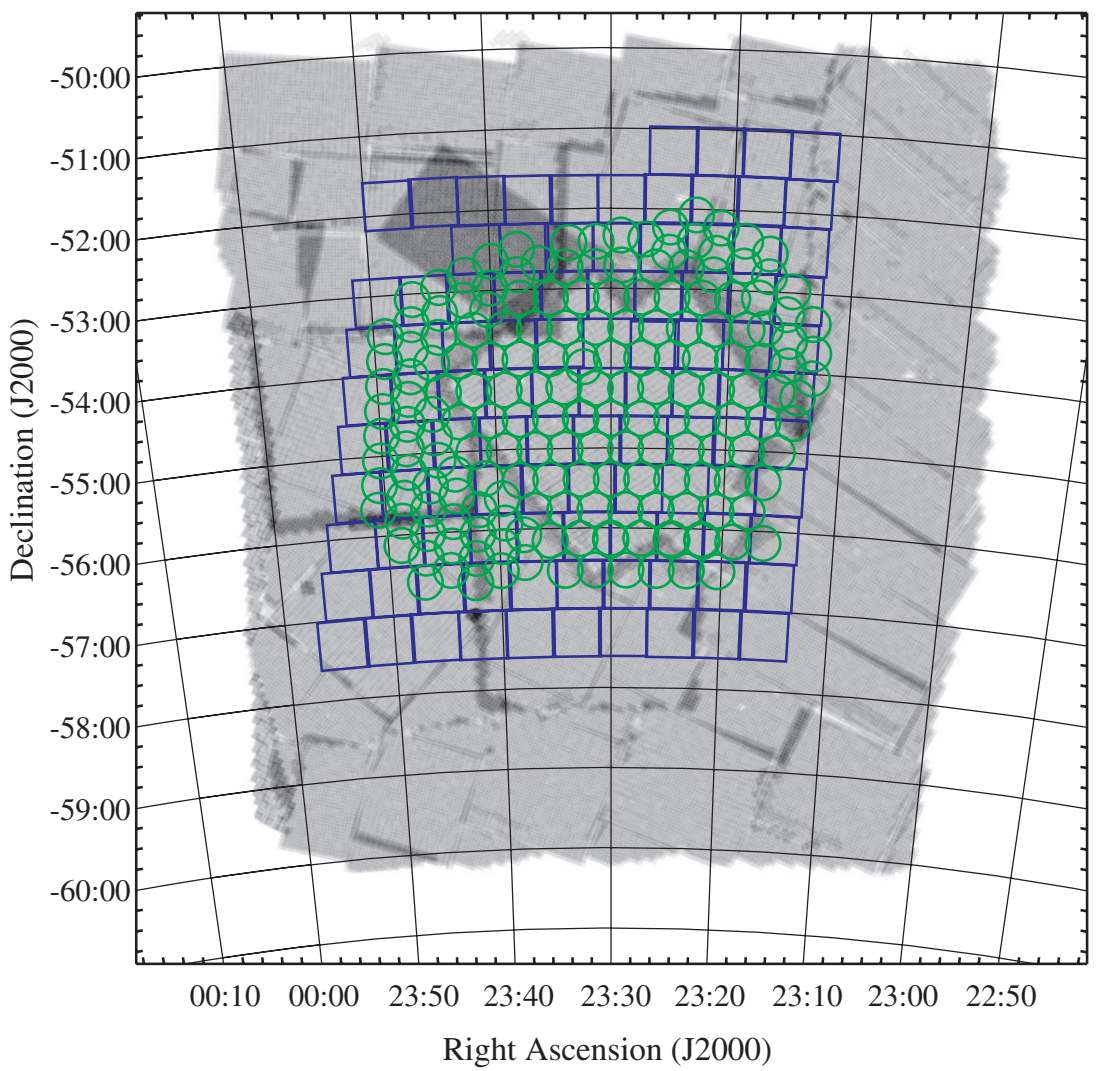

Figure 2. SSDF depth of coverage at $4.5 \mu \mathrm{m}$ (grayscale) including all observations taken through 2013 February. The linear stretch ranges from zero (white) to 15 (black). Most of the field is covered to the designed depth $(4 \times 30 \mathrm{~s})$. The coverage at $3.6 \mu \mathrm{m}$ is very similar to that shown here. Blue squares indicate tiles covered by griz observations from the Blanco Cosmology Survey (Desai et al. 2012). Green circles indicate XMM pointings from the XXL survey, covering a total of 25 deg ${ }^{2}$ to a depth of $10 \mathrm{ks}$

(A color version of this figure is available in the online journal.)

Table 1

Available Survey Data in SSDF

\begin{tabular}{|c|c|c|c|}
\hline $\begin{array}{l}\text { Waveband } \\
(\mu \mathrm{m})\end{array}$ & Origin & $\begin{array}{l}\text { Depth } \\
(5 \sigma)\end{array}$ & \\
\hline $0.5-0.9$ (ugriz) & Blanco Cosmology Survey & $\sim 1.0 \mu \mathrm{Jy}$ & L. Bleem et al., in preparation \\
\hline $1.35(J)$ & VISTA Hemisphere Survey & $12.0 \mu \mathrm{Jy}$ & R. McMahon et al. ${ }^{\mathrm{a}}$ \\
\hline $1.65(J)$ & VISTA Hemisphere Survey & $17.4 \mu \mathrm{Jy}$ & R. McMahon et al. ${ }^{\mathrm{a}}$ \\
\hline $2.20(K)$ & VISTA Hemisphere Survey & $30.2 \mu \mathrm{Jy}$ & R. McMahon et al. ${ }^{\mathrm{a}}$ \\
\hline 3.6 & Spitzer-SPT Deep Field & $7.0 \mu \mathrm{Jy}$ & This work \\
\hline 4.5 & Spitzer-SPT Deep Field & $9.4 \mu \mathrm{Jy}$ & This work \\
\hline 12 & WISE W3 & $1 \mathrm{mJy}$ & Wright et al. (2010) \\
\hline 22 & WISE W4 & $6 \mathrm{mJy}$ & Wright et al. (2010) \\
\hline 250 & Hershel/SPIRE & $10 \mathrm{mJy}$ & Holder et al. (2013) \\
\hline 350 & Hershel/SPIRE & $10 \mathrm{mJy}$ & Holder et al. (2013) \\
\hline 500 & Hershel/SPIRE & $10 \mathrm{mJy}$ & Holder et al. (2013) \\
\hline 1400 & SPT & $15 \mathrm{mJy}$ & Story et al. (2012) \\
\hline 2000 & SPT & $5 \mathrm{mJy}$ & Story et al. (2012) \\
\hline 3000 & SPT & $10 \mathrm{mJy}$ & Story et al. (2012) \\
\hline
\end{tabular}

Note. Depths of coverage for other surveys of the SSDF.

${ }^{a}$ http://www.eso.org/sci/observing/phase3/data_releases/vhs_dr1.html

relationship of the SSDF coverage to that of coincident surveys at other wavelengths is shown in Figures 2 and 3, is summarized in Table 1, and is described in detail below.

Their sensitivity to baryons in the intracluster medium makes X-ray observations particularly important for the study of galaxy clusters, and the XMM mission carried out a large-area survey specifically designed to be very sensitive to clusters. This is the XXL survey (Pierre et al. 2011), of which a $25 \mathrm{deg}^{2}$ portion is located in the SSDF. The XXL survey was performed in 2011-2013 with $10 \mathrm{ks}$ integrations per pointing, and the resulting cluster catalogs are now being confirmed via spectroscopic followup programs at the AngloAustralian Telescope, New Technology Telescope, and Very Large Telescope (M. Pierre et al., in preparation). The XXL survey is the largest deep-wide X-ray survey for galaxy clusters in existence, with an area five times greater than existing $X M M$ surveys and sensitivity sufficient to study clusters beyond $z=1$ down to masses of $\sim 10^{14} M_{\odot}$. The cross-comparison between 


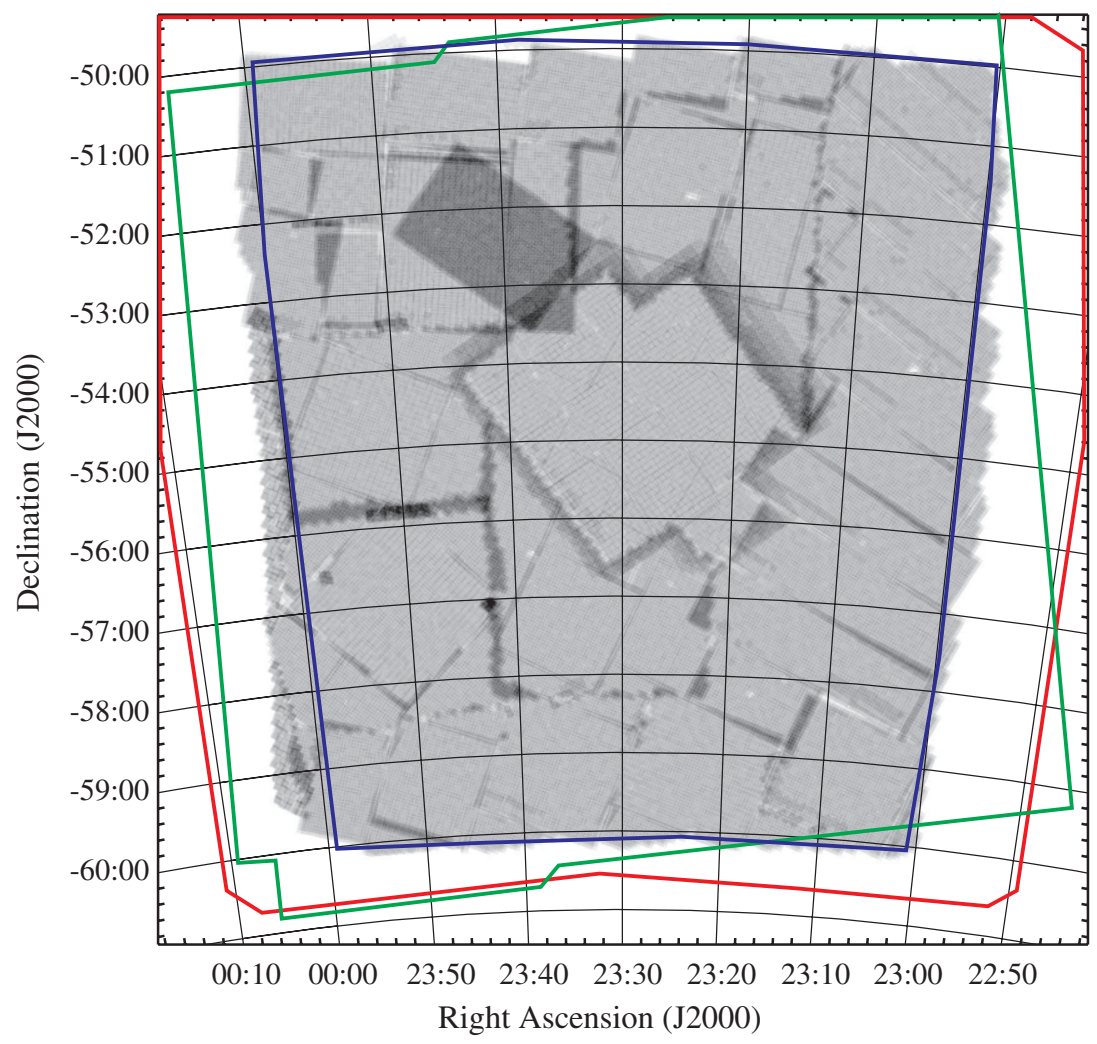

Figure 3. As Figure 2, but showing the boundaries of the coextensive coverage at long wavelengths. The extent of coverage obtained via SPT imaging at $1.4,2$, and $3 \mathrm{~mm}$ (the SPT deep field) is shown in red, while the Herschel/SPIRE far-infrared imaging is outlined in green, and the ATCA $16 \mathrm{~cm}$ imaging is outlined in blue.

(A color version of this figure is available in the online journal.)

the infrared and X-ray cluster catalogs will provide unique information about the purity and completeness of the samples as well as on the physics of high-redshift clusters.

The entire SSDF was also imaged at far-infrared wavelengths in 2011 with Herschel's Spectral and Photometric Imaging REceiver (SPIRE; Griffin et al. 2010). The SPIRE imaging reaches $1 \sigma$ depths of roughly $10 \mathrm{mJy}^{-1}$ beam ${ }^{-1}$ at 250,350 , and $500 \mu \mathrm{m}$. The SPIRE data will be very useful for studies of submillimeter galaxies and for cross-correlation studies involving cosmic microwave background lensing maps (e.g., Holder et al. 2013), and should probe ultraluminous infrared galaxy activity in galaxy clusters. Shallow infrared imaging is already available from the all-sky Wide-field Infrared Survey Explorer (WISE) survey (Wright et al. 2010), which reaches 1 and $6 \mathrm{mJy}$ at 12 and $22 \mu \mathrm{m}$, respectively. In the radio, the full field is being imaged with the Australia Telescope Compact Array (ATCA) at $16 \mathrm{~cm}$ down to $40 \mu \mathrm{Jy}_{\text {beam }}{ }^{-1}$ with a $7^{\prime \prime}$ beam.

In the visible/near-infrared regime, the SSDF already has extensive coverage from both completed and ongoing surveys. The Blanco Cosmology Survey (Desai et al. 2012; L. Bleem et al., in preparation) surveyed $\sim 30 \mathrm{deg}^{2}$ of the SSDF in the griz bands down to roughly $23 \mathrm{AB}$ mag $(10 \sigma)$. The ongoing Dark Energy Survey will obtain griz imaging down to approximately $24 \mathrm{AB}$ mag $(10 \sigma)$ over a large area that includes the entire SSDF. In the near-infrared, Data Release 1 of the VISTA Hemisphere Survey ${ }^{23}$ has already covered nearly the entire field in JHK to $5 \sigma$ limits of $21.2,20.8$, and $20.2 \mathrm{AB}$ mag.

Roughly $12 \mathrm{deg}^{2}$ in the center of the SSDF were observed with Spitzer/IRAC in Cycle 4 (PI: S. A. Stanford, PID 40370) with three dithered $30 \mathrm{~s}$ exposures. These observations were

\footnotetext{
${ }^{23}$ R. McMahon et al.,

http://www.eso.org/sci/observing/phase3/data_releases/vhs_dr1.html
}

incorporated into the SSDF and are hereafter referred to as the Spitzer-Blanco Cosmology Survey (S-BCS). Because it was obtained during Spitzer's cryogenic mission, the S-BCS dataset includes exposures at 5.8 and $8.0 \mu \mathrm{m}$, although those two longwavelength bands were not analyzed as part of the present work because they are significantly less sensitive than our new IRAC imaging.

The SSDF has already been fully covered at 95,150 , and $220 \mathrm{GHz}$ to $1 \sigma$ depths of 2,1 , and $3 \mathrm{mJy}^{\text {beam }}{ }^{-1}$, respectively by the SPT during its survey of more than $2500 \mathrm{deg}^{2}$ of the southern sky (Carlstrom et al. 2011; Story et al. 2012). The SPT provides an efficient means to detect high-redshift dusty galaxies (e.g., Vieira et al. 2013), but it is particularly effective for identifying galaxy clusters via the SZ effect (Sunyaev \& Zel'dovich 1972). The SZ selection technique is relatively insensitive to cluster redshift, so the SPT observations are capable of identifying galaxy clusters out to great distances (e.g., Reichardt et al. 2013). Over the next four years, even deeper observations by SPTpol (Austermann et al. 2012), which has already imaged this field to nearly twice the depth of the larger SPT survey (Story et al. 2012) will further improve the quality and depth of the millimeter data in this field. Its southern location means that it is well-placed for followup observations with the Atacama Large Millimeter/Submillimeter Array, and is a promising candidate for selection as one of the Large Synoptic Survey Telescope deep drilling fields.

\section{OBSERVATIONS AND DATA REDUCTION}

\subsection{Mapping Strategy}

The observing plan drew heavily on our team's prior experience with the IRAC Shallow Survey (Eisenhardt et al. 2004). The observations were arranged to account for and smoothly 
extend the pre-existing IRAC coverage from the $\mathrm{S}$-BCS $(3 \times 30 \mathrm{~s}$ depth over $\left.12 \mathrm{deg}^{2}\right)$. They also had to accommodate the position angle (P.A.) constraints of the two observing windows opening six months apart as well as the SSDF boundaries. The SSDF lies between declinations $-50 \mathrm{deg}$ and $-60 \mathrm{deg}$, and from right ascension $23 \mathrm{hr}$ to slightly east of $0 \mathrm{hr}$ (Figure 2). To accommodate these constraints, the SSDF was covered by Astronomical Observation Requests (AORs) having coverage footprints of various (sometimes irregular) shapes and sizes. When possible, standard IRAC grids were used to cover $\sim 1 \times 1 \mathrm{deg}^{2}$ areas in four passes, each pass obtaining a single $30 \mathrm{~s}$ exposure per position. Around the edges of and in between these grids, we designed AORs in fixed cluster mode so as to optimally cover irregular areas. Like the AORs covering areas having more regular shapes, these gerrymandered observations were also organized into groups of four single-pass AORs to cover each area, with a single $30 \mathrm{~s}$ exposure obtained in each pass. In any one area, the four AORs were obtained over the course of a two-day window. The goal was to achieve uniform coverage to the greatest extent possible.

Each AOR required 1-6 hr to execute, ensuring at least $1 \mathrm{hr}$ between successive observations of each sky position. For typical asteroid motions of $25^{\prime \prime} \mathrm{hr}^{-1}$, asteroids will move distances much greater than the IRAC point spread function (PSF) between AORs. The four observations of each sky position therefore allowed asteroids to be excluded from the final mosaics with standard outlier rejection techniques.

Our mapping strategy was to dither the exposures on small scales, and offset by one-third of an IRAC field of view between successive passes through each AOR group. This provided interpixel correlation information on both small and large scales. Our observing strategy is therefore very robust against bad rows/columns, large-scale cosmetic defects on the array, afterimages resulting from saturation due to bright stars, variations in the bias level, and the color dependence of the IRAC flat-field across the array (Quijada et al. 2004).

Although the four-AOR observations of specific areas were performed consecutively, spacecraft visibility constraints meant that coverage of the full SSDF had to be accumulated in separate campaigns spaced roughly six months apart. These took place in 2011 July-August, 2012 January-February, 2012 July-September, and 2012 December-2013 February. Because adjacent regions were inevitably observed at slightly different P.A.s, obvious and irregular coverage gaps were evident between adjacent mapping AOR sets as the observations accumulated. These were covered in the latter two epochs with clustermode AORs during which the exposures were placed to fill the coverage gaps as smoothly as possible given the spacecraft visibility limits and P.A.s. Like the AORs executed earlier, these irregular cluster-mode AORs were done in sets of single-pass rasters intended to accumulate a total of four dithered $30 \mathrm{~s}$ exposures everywhere.

\subsection{Data Reduction}

To the maximum extent possible, identical reduction procedures were applied to all SSDF and S-BCS data so as to ensure uniform data quality throughout the field. The data reduction was based on version S18.18.0 of the IRAC Corrected Basic Calibrated Data (cBCD) exposures for the first SSDF campaign; version S19.1.0 was used for all other, later campaigns. The cBCD data were used because some of the salient instrument artifacts (e.g., multiplexer bleed) are automatically corrected by the cBCD pipeline. Other artifacts (e.g., scattered light) are flagged in the $\mathrm{cBCD}$ pipeline-adjusted pixel masks for each frame. Both features of the $\mathrm{cBCD}$ data make them optimal for our purposes.

To remove slowly decaying residual images from unrelated observations of bright objects, all 3.6 and $4.5 \mu \mathrm{m}$ cBCD frames were object-masked and median-stacked on a per-AOR basis. The resulting stacked images (presumed to represent blank sky) were visually inspected and subtracted from individual cBCDs within each AOR. This created sky-subtracted versions of the cBCDs that were free of long-term residual images arising from prior observations of bright sources. Residual images with short decay times arising from observations of bright stars during the SSDF observations themselves were not addressed by this method, however. Pixels flagged as potentially contaminated with such residuals by the IRAC pipeline were masked. The sky-subtracted cBCDs were then examined individually and processed using custom software routines to correct columnpulldown effects associated with bright sources. The code, known as the "Warm-Mission Column Pulldown Corrector," is publicly available at the Spitzer Science Center. ${ }^{24}$

After these preliminaries, the SSDF exposures and the coincident IRAC imaging from the S-BCS were mosaicked with IRACproc (Schuster et al. 2006). IRACproc augments the capabilities of the standard IRAC reduction software (MOPEX) by calculating the spatial derivative for each image pixel and adjusting the clipping algorithm accordingly. Pixels where the derivative is low (in the field) are clipped more aggressively than are pixels where the spatial derivative is high (point sources). This avoids downward biasing of point source fluxes in the output mosaics that might otherwise occur because of the slightly undersampled IRAC PSF. The software was configured to automatically flag and reject cosmic ray hits based on pipelinegenerated masks together with the adjusted sigma-clipping algorithm for spatially coincident pixels.

The IRAC mosaics were organized into pairs of coextensive tiles each covering roughly $2 \times 1 \mathrm{deg}^{2}$ sub-fields at both 3.6 and $4.5 \mu \mathrm{m}$. A total of 46 tiles were required to cover the full SSDF. The IRAC coverage and the tile dimensions and locations are defined in Figure 4 and Table 2. By construction, the World Coordinate Systems of all SSDF tile pairs are tied to the coordinates of objects in the Two Micron All Sky Survey (2MASS) Point Source Catalog (Skrutskie et al. 2006), and are in perfect pixel-by-pixel registration.

The resulting 92 final mosaics/coverage map pairs (one pair per tile per band), are publicly available from the Exploration Science Programs website at the Spitzer Science Center. ${ }^{25}$ All SSDF mosaics were built with 0'.6 pixels and have units of $\mathrm{MJy} \mathrm{sr}^{-1}$.

\section{SOURCE EXTRACTION AND PHOTOMETRY}

\subsection{Source Identification}

We detected and photometered sources in the SSDF mosaics with SExtractor (ver. 2.8.6; Bertin \& Arnouts 1996). SExtractor is well-suited to the relatively sparse SSDF mosaics, where there are numerous source-free pixels available for robust sky background estimation. We adopted the SExtractor parameter settings shown in Table 3. The coverage maps constructed by IRACproc were used as detection weight images. Custom flag

\footnotetext{
24 http://ssc.spitzer.caltech.edu/archanaly/contributed/browse.html

25 http://irsa.ipac.caltech.edu/data/SPITZER/docs/spitzermission/ observingprograms/es/
} 


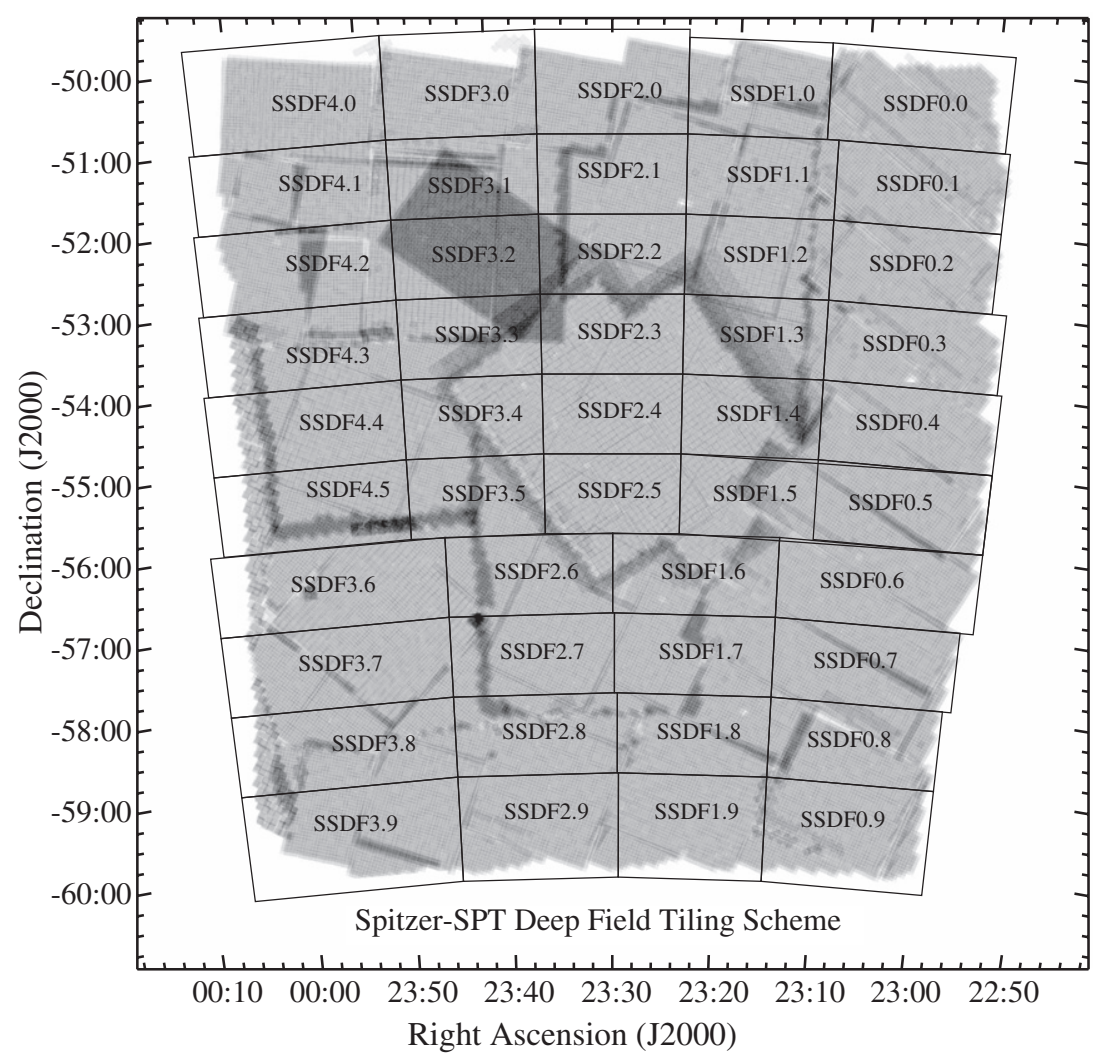

Figure 4. As Figure 2 but showing the tiling scheme used to define sub-regions of the SSDF in which mosaics were constructed as described in Section 3.2. The dark rectangle centered in tile SSDF3.2 at roughly $(\alpha, \delta)=(23: 40,-52: 30)$ is covered to $8 \times 30 \mathrm{~s}$, twice the nominal SSDF depth. The original $3 \times 30 \mathrm{~s}$ S-BCS observations (now covered to $4 \times 30 \mathrm{~s}$ depth) are apparent as the irregular region of uniform coverage centered on tile SSDF2.4.

images were constructed to identify and exclude all mosaic pixels covered by fewer than two exposures.

Photometry was measured within nine apertures having diameters ranging from $2^{\prime \prime}$ to $10^{\prime \prime}$ in $1^{\prime \prime}$ increments, plus two additional apertures of diameter $15^{\prime \prime}$ and $24^{\prime \prime}$, or eleven apertures in all. By comparing photometry measured in the $24^{\prime \prime}$ aperture (i.e., the aperture adopted by the Instrument Team as the fiducial photometric aperture for IRAC) to that in all other apertures for well-detected, isolated sources, we obtained empirical estimates of the aperture corrections, which are given in Table 4. We also obtained MAG_AUTO magnitudes, for which SExtractor measures fluxes interior to elliptical apertures having sizes and orientations determined using the second-order moments of the light distribution measured above the isophotal threshold. We compared the MAG_AUTO photometry to the corrected-to-total aperture magnitudes and found that the MAG_AUTO estimates were systematically fainter by roughly $0.05 \mathrm{mag}$. In other words, the corrected aperture magnitudes are consistent with each other, but the MAG_AUTO measurements are 0.05 mag fainter on average.

We used SExtractor in dual-image mode. In this configuration, sources are detected, their centers are located, and their apertures are defined in one image, and subsequently photometry is carried out in another image using those pre-established apertures and source centroids. The dual-image approach forces SExtractor to measure the emission from all sources over identical areas in both bands, ensuring that the resulting photometry will yield accurate source colors. SExtractor was configured to define a source as a set of four or more connected pixels each lying $0.5 \sigma$ above the estimated background. We first used the $4.5 \mu \mathrm{m}$ mosaics as the detection images; both the 3.6 and $4.5 \mu \mathrm{m}$ mosaics were used in turn as the photometry images. The process was followed in all 46 tiles covering the SSDF, resulting in 46 pairs of single-band catalogs.

Because the source extraction was performed in dual-image mode, the separate 3.6 and $4.5 \mu \mathrm{m}$ SExtractor catalog pairs generated for each of the two selection bands were in line-by-line registration. We generated band-merged catalogs by combining photometry from these catalog pairs for all SSDF tiles. The band-merged, tile-based catalogs were trimmed to exclude overlapping regions at the tile boundaries given in Table 2, and all aperture photometry was corrected to total magnitudes using the empirical aperture corrections from Table 4. A full-field catalog covering the entire SSDF was then created by combining the trimmed, band-merged, aperture-corrected photometry from all tiles. We chose to include the corrected $4^{\prime \prime}$ and $6^{\prime \prime}$ diameter aperture magnitudes in the final catalogs, along with the SExtractor MAG_AUTO magnitudes.

Finally, the above process was repeated using the $3.6 \mu \mathrm{m}$ mosaics as the detection images. The result is a pair of fullfield band-merged SSDF catalogs-one selected at $3.6 \mu \mathrm{m}$, and another selected at $4.5 \mu \mathrm{m}$.

\subsection{Survey Depth, Completeness, and Astrometric Reliability}

\subsubsection{Survey Depth and Completeness Estimation}

We used a Monte Carlo approach to estimate the survey completeness and sensitivity by placing numerous simulated sources in the SSDF mosaics at random locations and then photometering them in an identical manner to that used for the original mosaics. Specifically, we inserted simulated objects in both the 3.6 and $4.5 \mu \mathrm{m}$ mosaics for five different SSDF tiles 
Table 2

SPT-Spitzer Tile Definitions

\begin{tabular}{|c|c|c|}
\hline Tile & $\begin{array}{l}\text { Right Ascension Range } \\
\text { (deg, J2000) }\end{array}$ & $\begin{array}{l}\text { Declination Range } \\
\quad(\text { deg, J2000) }\end{array}$ \\
\hline SSDF0.0 & $\alpha \leqslant 348.23$ & $-49.8 \geqslant \delta>-51.0$ \\
\hline SSDF0.1 & $\alpha \leqslant 348.00$ & $-51.0 \geqslant \delta>-52.0$ \\
\hline SSDF0.2 & $\alpha \leqslant 348.00$ & $-52.0 \geqslant \delta>-53.0$ \\
\hline SSDF0.3 & $\alpha \leqslant 348.00$ & $-53.0 \geqslant \delta>-54.0$ \\
\hline SSDF0.4 & $\alpha \leqslant 348.00$ & $-54.0 \geqslant \delta>-55.0$ \\
\hline SSDF0.5 & $\alpha \leqslant 348.00$ & $-55.0 \geqslant \delta>-56.0$ \\
\hline SSDF0.6 & $\alpha \leqslant 348.75$ & $-56.0 \geqslant \delta>-57.0$ \\
\hline SSDF0.7 & $\alpha \leqslant 348.75$ & $-57.0 \geqslant \delta>-58.0$ \\
\hline SSDF0.8 & $\alpha \leqslant 348.75$ & $-58.0 \geqslant \delta>-59.0$ \\
\hline SSDF0.9 & $\alpha \leqslant 348.75$ & $-59.0 \geqslant \delta>-60.2$ \\
\hline SSDF1.0 & $348.23<\alpha \leqslant 351.00$ & $-49.8 \geqslant \delta>-51.0$ \\
\hline SSDF1.1 & $348.00<\alpha \leqslant 351.00$ & $-51.0 \geqslant \delta>-52.0$ \\
\hline SSDF1.2 & $348.00<\alpha \leqslant 351.00$ & $-52.0 \geqslant \delta>-53.0$ \\
\hline SSDF1.3 & $348.00<\alpha \leqslant 351.00$ & $-53.0 \geqslant \delta>-54.0$ \\
\hline SSDF1.4 & $348.00<\alpha \leqslant 351.00$ & $-54.0 \geqslant \delta>-55.0$ \\
\hline SSDF1.5 & $348.00<\alpha \leqslant 351.00$ & $-55.0 \geqslant \delta>-56.0$ \\
\hline SSDF1.6 & $348.75<\alpha \leqslant 352.49$ & $-56.0 \geqslant \delta>-57.0$ \\
\hline SSDF1.7 & $348.75<\alpha \leqslant 352.45$ & $-57.0 \geqslant \delta>-58.0$ \\
\hline SSDF1.8 & $348.75<\alpha \leqslant 352.38$ & $-58.0 \geqslant \delta>-59.0$ \\
\hline SSDF1.9 & $348.75<\alpha \leqslant 352.35$ & $-59.0 \geqslant \delta>-60.2$ \\
\hline SSDF2.0 & $351.00<\alpha \leqslant 354.00$ & $-49.8 \geqslant \delta>-51.0$ \\
\hline SSDF2.1 & $351.00<\alpha \leqslant 354.00$ & $-51.0 \geqslant \delta>-52.0$ \\
\hline SSDF2.2 & $351.00<\alpha \leqslant 354.00$ & $-52.0 \geqslant \delta>-53.0$ \\
\hline SSDF2.3 & $351.00<\alpha \leqslant 354.00$ & $-53.0 \geqslant \delta>-54.0$ \\
\hline SSDF2.4 & $351.00<\alpha \leqslant 354.00$ & $-54.0 \geqslant \delta>-55.0$ \\
\hline SSDF2.5 & $351.00<\alpha \leqslant 354.00$ & $-55.0 \geqslant \delta>-56.0$ \\
\hline SSDF2.6 & $352.49<\alpha \leqslant 356.25$ & $-56.0 \geqslant \delta>-57.0$ \\
\hline SSDF2.7 & $352.45<\alpha \leqslant 356.25$ & $-57.0 \geqslant \delta>-58.0$ \\
\hline SSDF2.8 & $352.38<\alpha \leqslant 356.25$ & $-58.0 \geqslant \delta>-59.0$ \\
\hline SSDF2.9 & $352.35<\alpha \leqslant 356.25$ & $-59.0 \geqslant \delta>-60.2$ \\
\hline SSDF3.0 & $354.00<\alpha \leqslant 357.00$ & $-49.8 \geqslant \delta>-51.0$ \\
\hline SSDF3.1 & $354.00<\alpha \leqslant 357.00$ & $-51.0 \geqslant \delta>-52.0$ \\
\hline SSDF3.2 & $354.00<\alpha \leqslant 357.00$ & $-52.0 \geqslant \delta>-53.0$ \\
\hline SSDF3.3 & $354.00<\alpha \leqslant 357.00$ & $-53.0 \geqslant \delta>-54.0$ \\
\hline SSDF3.4 & $354.00<\alpha \leqslant 357.00$ & $-54.0 \geqslant \delta>-55.0$ \\
\hline SSDF3.5 & $354.00<\alpha \leqslant 357.00$ & $-55.0 \geqslant \delta>-56.0$ \\
\hline SSDF3.6 & $356.25<\alpha ; \alpha<2.0$ & $-56.0 \geqslant \delta>-57.0$ \\
\hline SSDF3.7 & $356.25<\alpha ; \alpha<2.0$ & $-57.0 \geqslant \delta>-58.0$ \\
\hline SSDF3.8 & $356.25<\alpha ; \alpha<2.0$ & $-58.0 \geqslant \delta>-59.0$ \\
\hline SSDF3.9 & $356.25<\alpha ; \alpha<2.0$ & $-59.0 \geqslant \delta>-61.2$ \\
\hline SSDF4.0 & $357.00<\alpha ; \alpha<2.0$ & $-49.8 \geqslant \delta>-51.0$ \\
\hline SSDF4.1 & $357.00<\alpha ; \alpha<2.0$ & $-51.0 \geqslant \delta>-52.0$ \\
\hline SSDF4.2 & $357.00<\alpha ; \alpha<2.0$ & $-52.0 \geqslant \delta>-53.0$ \\
\hline SSDF4.3 & $357.00<\alpha ; \alpha<2.0$ & $-53.0 \geqslant \delta>-54.0$ \\
\hline SSDF4.4 & $357.00<\alpha ; \alpha<2.0$ & $-54.0 \geqslant \delta>-55.0$ \\
\hline SSDF4.5 & $357.00<\alpha ; \alpha<2.0$ & $-55.0 \geqslant \delta>-56.0$ \\
\hline
\end{tabular}

Note. The locations and dimensions of sub-regions (tiles) in which the SSDF IRAC data were reduced in pixel-pixel registration.

(labeled SSDF1.6, 2.4, 2.6, 3.2, and 3.5 in Figure 4) chosen as representative of the range of IRAC depths of coverage that we obtained. The total area in which the simulated sources were inserted and subsequently photometered therefore samples roughly $10.6 \mathrm{deg}^{2}$ of the total survey field.

The simulated sources were randomly assigned magnitudes between 10 and 21 Vega mag. Hundreds of simulated sources in this range were simultaneously placed at random locations in each of the five tiles employed for this purpose. The number of simulated sources inserted at one time was restricted to a small percentage of the total number of objects apparent in the field, so as to avoid artificially induced source confusion effects. Nonetheless, because the simulated sources were allowed to fall
Table 3

SSDF SExtractor Parameter Settings

\begin{tabular}{lc}
\hline \hline Parameter & Setting \\
\hline DETECT_MINAREA (pixel) & 4 \\
DETECT_THRESH (sigma) & 0.5 \\
FILTER & gauss_3.0_7×7 \\
DEBLEND_NTHRESH & 64 \\
DEBLEND_MINCONT & 0.0001 \\
BACK_SIZE (pixel) & 128 \\
BACK_FILTERSIZE & 3 \\
BACKPHOTO_TYPE & GLOBAL
\end{tabular}

Notes. Parameter settings used to identify and photometer sources identically in both of the SSDF IRAC bands. The only SExtractor settings that differed in the two bands were SEEING_FWHM and MAG_ZERO.SEEING_FWHM was set to $1^{\prime \prime} .66$ and $1^{\prime \prime} .72$ in the 3.6 and $4.5 \mu \mathrm{m}$ mosaics, respectively. MAG_ZERO was set to $18.789(3.6 \mu \mathrm{m})$ and 18.316 Vega mag $(4.5 \mu \mathrm{m})$.

Table 4

SSDF Aperture Corrections

\begin{tabular}{lcc}
\hline $\begin{array}{l}\text { Diameter } \\
(\operatorname{arcsec})\end{array}$ & $\begin{array}{c}3.6 \mu \mathrm{m} \\
(\mathrm{mag})\end{array}$ & $\begin{array}{c}4.5 \mu \mathrm{m} \\
(\mathrm{mag})\end{array}$ \\
\hline $2^{\prime \prime}$ & -1.13 & -1.07 \\
$3^{\prime \prime}$ & -0.58 & -0.56 \\
$4^{\prime \prime}$ & -0.33 & -0.33 \\
$5^{\prime \prime}$ & -0.22 & -0.20 \\
$6^{\prime \prime}$ & -0.16 & -0.14 \\
$7^{\prime \prime}$ & -0.13 & -0.11 \\
$8^{\prime \prime}$ & -0.11 & -0.09 \\
$9^{\prime \prime}$ & -0.09 & -0.08 \\
$10^{\prime \prime}$ & -0.08 & -0.07 \\
$15^{\prime \prime}$ & -0.04 & -0.03 \\
\hline
\end{tabular}

Notes. Aperture corrections (magnitudes) derived from comparisons of photometry in the SSDF apertures to that measured in the fiducial IRAC aperture (diameter $24^{\prime \prime}$ ). These corrections are consistent with those tabulated in the IRAC Instrument Handbook. All photometry compiled in the SSDF catalogs presented in this work has been aperture-corrected to total magnitudes using these values.

anywhere in their respective tiles, including atop real sources, the simulations do account realistically for the effects of source confusion. The process was iterated, so that a total population of 20,000 simulated sources was ultimately analyzed in each of the 0.5 mag wide bins we constructed to span the magnitude range we considered.

After processing the modified mosaics with SExtractor in exactly the same way as was done for the original mosaics, the resulting catalogs were compared to determine the completeness as a function of magnitude. The comparison was performed in catalog space with MAG_AUTO magnitudes using a simple position-matching criterion. An additional constraint was imposed, requiring that a valid detection of a simulated source had to yield a measured magnitude that fell within 0.5 mag of its a priori known magnitude to account for source confusion. This procedure was repeated in both bands for all five tiles tested. The results are given in Table 5 and shown in Figure 5.

The SSDF catalogs include only sources with aperturecorrected (total) magnitudes brighter than the achieved sensitivity levels in at least one of the two SSDF bands. We defined the SSDF sensitivity as the magnitude at which the empirical uncertainty in the SExtractor-estimated fluxes reached 
Table 5

SSDF Completeness

\begin{tabular}{|c|c|c|c|c|}
\hline \multirow{2}{*}{$\begin{array}{l}\text { Mag } \\
\text { (Vega) }\end{array}$} & \multicolumn{2}{|c|}{$3.6 \mu \mathrm{m}$} & \multicolumn{2}{|c|}{$4.5 \mu \mathrm{m}$} \\
\hline & Completeness & Unc. & Completeness & Unc. \\
\hline 9.5 & $\ldots$ & $\ldots$ & 0.999 & 0.002 \\
\hline 10.0 & 0.998 & 0.002 & 0.998 & 0.002 \\
\hline 10.5 & 0.997 & 0.002 & 0.998 & 0.001 \\
\hline 11.0 & 0.997 & 0.001 & 0.997 & 0.003 \\
\hline 11.5 & 0.996 & 0.001 & 0.996 & 0.003 \\
\hline 12.0 & 0.993 & 0.003 & 0.995 & 0.002 \\
\hline 12.5 & 0.992 & 0.003 & 0.991 & 0.004 \\
\hline 13.0 & 0.988 & 0.005 & 0.990 & 0.003 \\
\hline 13.5 & 0.985 & 0.005 & 0.987 & 0.004 \\
\hline 14.0 & 0.982 & 0.006 & 0.981 & 0.004 \\
\hline 14.5 & 0.976 & 0.007 & 0.974 & 0.005 \\
\hline 15.0 & 0.968 & 0.006 & 0.968 & 0.002 \\
\hline 15.5 & 0.955 & 0.007 & 0.949 & 0.006 \\
\hline 16.0 & 0.933 & 0.007 & 0.932 & 0.005 \\
\hline 16.5 & 0.910 & 0.004 & 0.90 & 0.01 \\
\hline 17.0 & 0.88 & 0.01 & 0.84 & 0.01 \\
\hline 17.5 & 0.80 & 0.04 & 0.72 & 0.03 \\
\hline 18.0 & 0.71 & 0.03 & 0.55 & 0.03 \\
\hline 18.5 & 0.55 & 0.03 & 0.39 & 0.03 \\
\hline 19.0 & 0.39 & 0.03 & 0.27 & 0.02 \\
\hline 19.5 & 0.27 & 0.02 & 0.19 & 0.02 \\
\hline 20.0 & 0.18 & 0.01 & 0.11 & 0.01 \\
\hline 20.5 & 0.10 & 0.01 & $\ldots$ & $\ldots$ \\
\hline
\end{tabular}

Notes. Completeness estimates and uncertainties for the SSDF at 3.6 and $4.5 \mu \mathrm{m}$. Uncertainties are empirical estimates based on variations in completeness measured in five separate tiles. The completeness is unity at magnitudes brighter than those listed, although such sources will be saturated in the SSDF mosaics.

approximately the $5 \sigma$ level $(0.2 \mathrm{mag})$. This occurred at $[3.6]=19.0 \mathrm{mag}(7.0 \mu \mathrm{Jy})$ and $[4.5]=18.2 \mathrm{mag}(9.4 \mu \mathrm{Jy})$ for $4^{\prime \prime}$ diameter apertures, similar to the sensitivity reported by Eisenhardt et al. (2004) for the first IRAC survey of Boötes: [3.6] = $19.1 \mathrm{mag}$ and [4.5] = $18.3 \mathrm{mag}$. The SSDF thus achieves $5 \sigma$ sensitivities similar to those predicted by the online Sensitivity Performance Estimation Tool (SENS-PET), which were $19.2 \mathrm{mag}$ and [4.5] $=18.6 \mathrm{mag}$ assuming low-background conditions.

\subsubsection{Astrometric Reliability}

To estimate the accuracy of the SSDF astrometry, we compared SSDF IRAC positions of bright but unsaturated sources to those in the 2MASS Point Source Catalog (Skrutskie et al. 2006). We performed a search within $1^{\prime \prime}$ of the positions of IRAC sources to identify their 2MASS counterparts. The distributions of coordinate offsets for the 3.6 and $4.5 \mu \mathrm{m}$ sources are shown in Figure 6. The astrometric discrepancies are small compared to the size of a SSDF pixel: the mean difference (SSDF-2MASS) was just -0 '. $15 \pm 0$ '.26 in right ascension and 0 '.03 \pm 0 '.23 in declination. The total radial uncertainties are therefore $0{ }^{\prime} .15(1 \sigma)$ relative to 2MASS. This is about one-fourth of an SSDF mosaic pixel, and less than one-tenth of the FWHM of the IRAC PSF in either band. This is comparable to the astrometric precision obtained in other Spitzer/IRAC surveys, e.g., SDWFS (Ashby et al. 2009).

\section{SSDF CATALOGS}

Both versions of the band-merged SSDF catalogs are presented here, one for each of the two selection bands (3.6 and $4.5 \mu \mathrm{m})$. The catalogs contain a total of $4.7 \times 10^{6}$ and $3.5 \times 10^{6}$

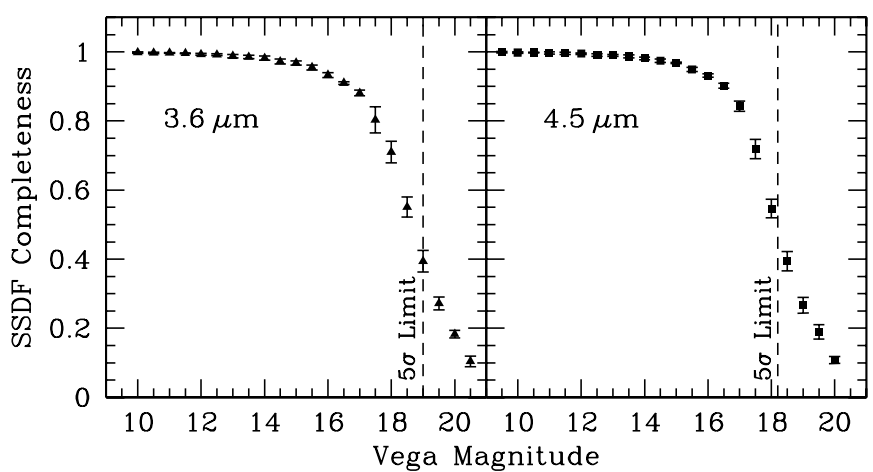

Figure 5. Recovered fraction of simulated SSDF sources as a function of input magnitude, based on the simulations described in Section 4.2.

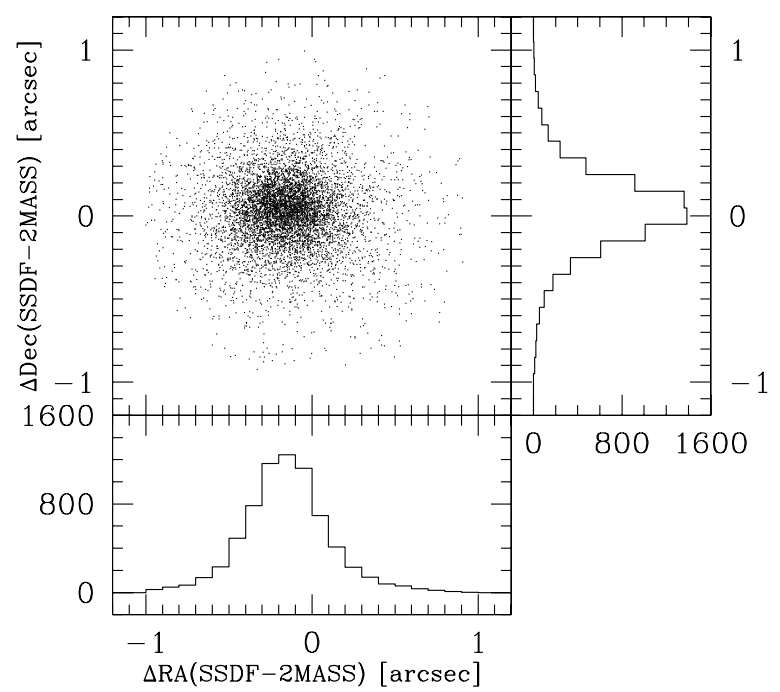

Figure 6. Coordinate offsets measured for IRAC-detected sources also detected in the 2MASS Point Source Catalog. The mean differences are $-00^{\prime \prime} 15 \pm 0$ '. 26 in right ascension and $0.203 \pm 0$ '.23 in declination, giving a total radial uncertainty of just $0^{\prime \prime} 15(1 \sigma)$ relative to 2MASS, or less than one-tenth of the FWHM of the IRAC point spread function at $3.6 \mu \mathrm{m}$.

$3.6 \mu \mathrm{m}$ and $4.5 \mu \mathrm{m}$ selected sources, respectively, down to the $5 \sigma$ detection thresholds. The formats of the two catalogs are identical and are defined in Table 6. All aperture magnitudes in both catalogs have been corrected-to-total magnitudes using the aperture corrections given in Table 4 . The catalogs also contain MAG_AUTO total magnitude estimates. All photometric estimates are provided with $1 \sigma$ uncertainty estimates generated by SExtractor, and are also given as flux densities in units of $\mu \mathrm{Jy}$. In addition to the photometry, the SSDF catalog provides a number of SExtractor-derived descriptors for each source as measured in the selection band, 3.6 or $4.5 \mu \mathrm{m}$ as appropriate (Table 6).

\section{DISCUSSION}

The measured colors of celestial sources reflect their underlying nature, albeit after being folded through the detection/selection process. The IRAC colors for all sources listed in the two SSDF catalogs are shown in Figure 7. The color distributions are broadly consistent with what is seen typically with IRAC at these flux levels, e.g., Ashby et al. (2009). For example, the faintest SSDF sources (those fainter than [3.6] = $[4.5]=17.5$ Vega mag) are systematically and significantly 
Table 6

Spitzer-SPT Photometry Catalog Column Definitions

\begin{tabular}{|c|c|c|c|}
\hline Column & Parameter & Description & Units \\
\hline 1 & TILE & SSDF sub-tile of origin & \\
\hline 2 & X_IMAGE & Object position along $x$ & pixel \\
\hline 3 & Y_IMAGE & Object position along $y$ & pixel \\
\hline 4 & ALPHA_J2000 & Right ascension of barycenter (J2000) & deg \\
\hline 5 & DELTA_J2000 & Declination of barycenter (J2000) & deg \\
\hline 6 & KRON_RADIUS & Kron apertures in units of $\mathrm{A}$ or $\mathrm{B}$ & \\
\hline 7 & BACKGROUND & Background at centroid position & count \\
\hline 8 & FLUX_RADIUS & Fraction-of-light radii & pixel \\
\hline 9 & ALPHAPEAK_J2000 & Right ascension of brightest pixel (J2000) & $\operatorname{deg}$ \\
\hline 10 & DELTAPEAK_J2000 & Declination of brightest pixel (J2000) & $\operatorname{deg}$ \\
\hline 11 & X2_IMAGE & Variance along $x$ & pixel $^{2}$ \\
\hline 12 & Y2_IMAGE & Variance along $y$ & pixel $^{2}$ \\
\hline 13 & XY_IMAGE & Covariance between $x$ and $y$ & pixel $^{2}$ \\
\hline 14 & A_IMAGE & Profile RMS along major axis & pixel \\
\hline 15 & B_IMAGE & Profile RMS along minor axis & pixel \\
\hline 16 & THETA_IMAGE & Position angle $(\mathrm{CCW} / x)$ & deg \\
\hline 17 & A_WORLD & Profile RMS along major axis (world units) & deg \\
\hline 18 & B_WORLD & Profile RMS along minor axis (world units) & deg \\
\hline 19 & THETA_WORLD & Position angle (CCW/world- $x)$ & $\operatorname{deg}$ \\
\hline 20 & CLASS_STAR & $\mathrm{S} / \mathrm{G}$ classifier output & \\
\hline 21 & FLAGS & SExtractor flags & \\
\hline \multicolumn{4}{|c|}{ The following 12 quantities correspond to IRAC $3.6 \mu \mathrm{m}$ measurements } \\
\hline 22 & MAG_AUTO & Kron-like elliptical aperture magnitude & Vega mag \\
\hline 23 & MAGERR_AUTO & RMS error for AUTO magnitude & Vega mag \\
\hline 24 & MAG_APER & $4^{\prime \prime}$ diameter aperture magnitude, corrected & Vega mag \\
\hline 25 & MAGERR_APER & $4^{\prime \prime}$ diameter aperture magnitude uncertainty & Vega mag \\
\hline 26 & MAG_APER & $6^{\prime \prime}$ diameter aperture magnitude, corrected & Vega mag \\
\hline 27 & MAGERR_APER & $6^{\prime \prime}$ diameter aperture magnitude uncertainty & Vega mag \\
\hline 28 & FLUX_AUTO & Kron-like elliptical aperture flux & $\mu \mathrm{Jy}$ \\
\hline 29 & FLUXERR_AUTO & RMS error for AUTO flux & $\mu \mathrm{Jy}$ \\
\hline 30 & FLUX_APER & $4^{\prime \prime}$ diameter aperture flux, corrected & $\mu \mathrm{Jy}$ \\
\hline 31 & FLUXERR_APER & $4^{\prime \prime}$ diameter aperture flux, uncertainty & $\mu \mathrm{Jy}$ \\
\hline 32 & FLUX_APER & $6^{\prime \prime}$ diameter aperture flux, corrected & $\mu \mathrm{Jy}$ \\
\hline 33 & FLUXERR_APER & $6^{\prime \prime}$ diameter aperture flux, uncertainty & $\mu \mathrm{Jy}$ \\
\hline \multicolumn{4}{|c|}{ The following 12 quantities correspond to IRAC $4.5 \mu \mathrm{m}$ measurements } \\
\hline 34 & MAG_AUTO & Kron-like elliptical aperture magnitude & Vega mag \\
\hline 35 & MAGERR_AUTO & RMS error for AUTO magnitude & Vega mag \\
\hline 36 & MAG_APER & $4^{\prime \prime}$ diameter aperture magnitude, corrected & Vega mag \\
\hline 37 & MAGERR_APER & $4^{\prime \prime}$ diameter aperture magnitude uncertainty & Vega mag \\
\hline 38 & MAG_APER & $6^{\prime \prime}$ diameter aperture magnitude, corrected & Vega mag \\
\hline 39 & MAGERR_APER & $6^{\prime \prime}$ diameter aperture magnitude uncertainty & Vega mag \\
\hline 40 & FLUX_AUTO & Kron-like elliptical aperture magnitude & $\mu \mathrm{Jy}$ \\
\hline 41 & FLUXERR_AUTO & RMS error for AUTO magnitude & $\mu \mathrm{Jy}$ \\
\hline 42 & FLUX_APER & $4^{\prime \prime}$ diameter aperture flux, corrected & $\mu \mathrm{Jy}$ \\
\hline 43 & FLUXERR_APER & $4^{\prime \prime}$ diameter aperture flux uncertainty & $\mu \mathrm{Jy}$ \\
\hline 44 & FLUX_APER & $6^{\prime \prime}$ diameter aperture flux, corrected & $\mu \mathrm{Jy}$ \\
\hline 45 & FLUXERR_APER & $6^{\prime \prime}$ diameter aperture flux uncertainty & $\mu \mathrm{Jy}$ \\
\hline
\end{tabular}

Notes. The column definitions for both Spitzer-SPT Deep Field catalogs. All columns except Column 1 contain quantities output by SExtractor (Bertin \& Arnouts 1996); Column 1 specifies the mosaic sub-fields (tiles; see Figure 4 and Table 2) processed individually by SExtractor to generate the photometric measurements tabulated in these catalogs.

(This table is available in its entirety in a machine-readable form in the online journal. A portion is shown here for guidance regarding its form and content.)

redder than brighter SSDF sources. This is because the surface density of relatively red extragalactic sources increases quickly below $17 \mathrm{mag}$, while the contribution from Galactic stars, which are relatively blue in the IRAC bands, flattens out (e.g., Fazio et al. 2004a).

The differential IRAC number counts in the SSDF are given in Table 7 and Figure 8, after applying appropriate corrections for incompleteness based on the empirical estimates in Table 5 .
Although the SSDF catalogs contain many sources brighter than $10 \mathrm{mag}$, these are not shown in the source counts because they are saturated in the IRAC mosaics. Nonetheless the SSDF counts are broadly consistent with counts measured earlier by, e.g., Ashby et al. (2009) and Fazio et al. (2004a), over the magnitude range covered by the SSDF catalogs. At bright flux levels, the SSDF counts are slightly elevated with respect to SDWFS. We have examined the SSDF mosaics at the locations of sources 


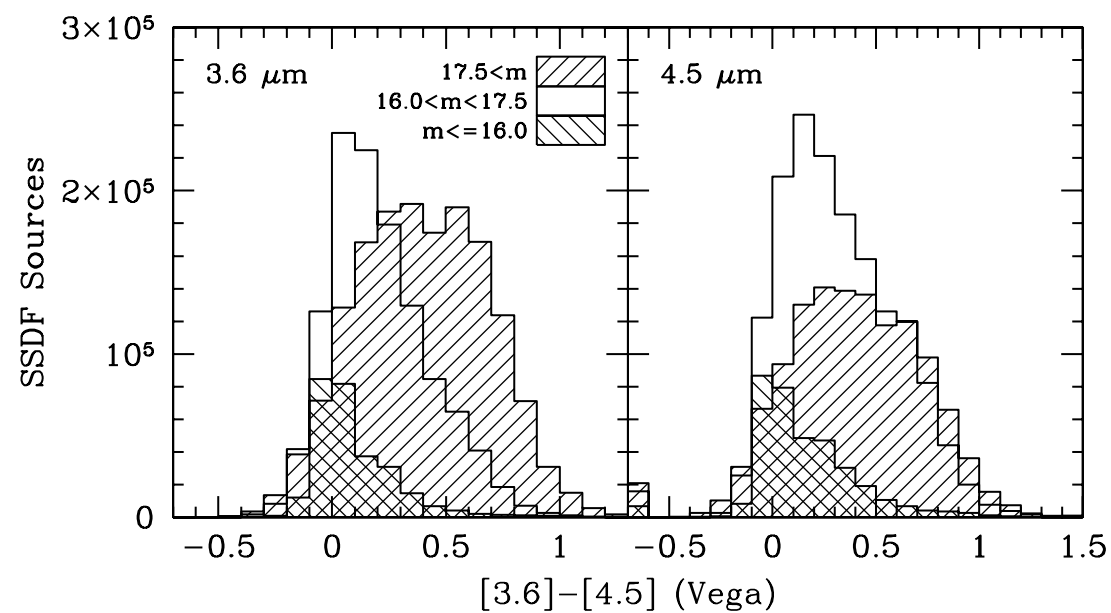

Figure 7. Color distributions of SSDF sources detected with greater than $5 \sigma$ significance in both IRAC bands. All colors are measured from aperture-corrected $4^{\prime \prime}$ diameter aperture photometry. Left panel: colors for SSDF sources selected at $3.6 \mu \mathrm{m}$. The larger hatched histogram corresponds to the faintest sources, i.e., those fainter than [3.6] $=17.5 \mathrm{mag}$. The brightest sources (those brighter than [3.6] $=16 \mathrm{mag}$ ) are indicated with the smaller hatched histogram. Those of intermediate brightness are indicated with the open histogram. Right panel: as for the left panel, but for SSDF sources selected at $4.5 \mu \mathrm{m}$.

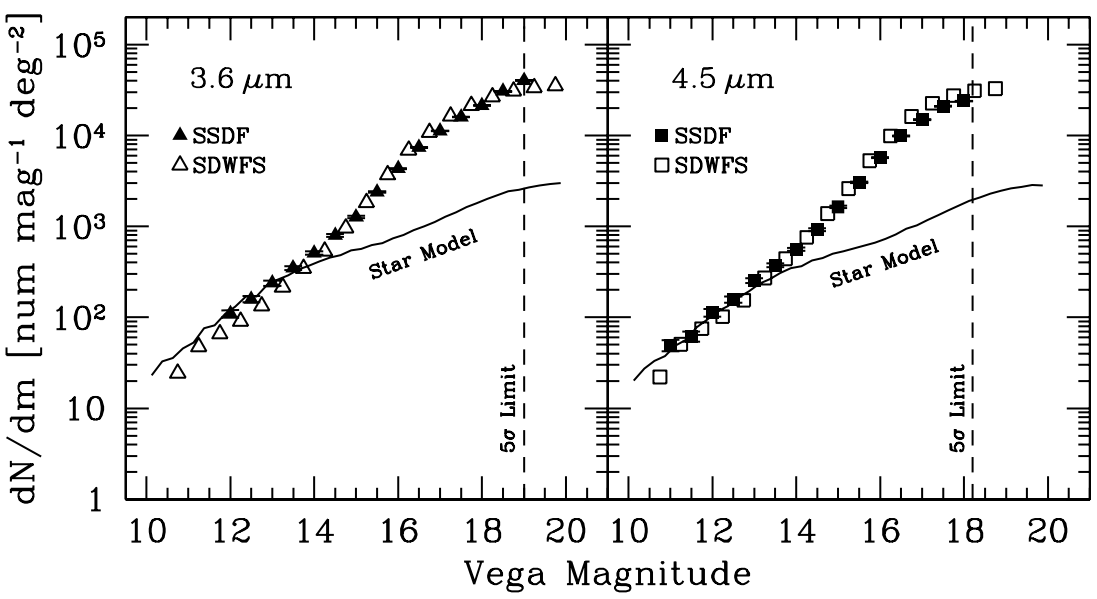

Figure 8. Differential source counts for the SSDF in the two IRAC bands. The $3.6 \mu \mathrm{m}$ counts (left panel, solid triangles) are taken from the $3.6 \mu \mathrm{m}$ selected SSDF catalog, while the $4.5 \mu \mathrm{m}$ counts (right panel, solid squares) are taken from the $4.5 \mu \mathrm{m}$ selected catalog. All counts shown here are based on SExtractor MAG_AUTO estimates for unsaturated sources and are corrected for the effects of incompleteness using the empirical estimates given in Table 5. The solid lines indicate the expected counts arising from Milky Way stars along the line of sight through the center of the SSDF at $(\alpha, \delta)=(23: 30,-55: 00)$ based on the DIRBE Faint Source Model at 3.5 and $4.9 \mu \mathrm{m}$ in the left and right panels, respectively (Arendt et al. 1998; Wainscoat et al. 1992; Cohen 1993, 1994, 1995). The SSDF counts are similar to those measured in the narrower but more sensitive Spitzer Deep, Wide Field Survey (open symbols; Ashby et al. 2009), as discussed in Section 6.

in the affected magnitude ranges, and find that virtually all are pointlike. This is consistent with a picture in which these sources are due to Milky Way stars, seen along a line of sight that is closer in both latitude and longitude $(\ell, b)=(325,-58)$ to the Galactic center than is $\operatorname{SDWFS}(\ell, b)=(60,+67)$. This is borne out by the consistency between the DIRBE model Milky Way star counts and the bright IRAC SSDF counts shown in Figure 8.

In the $30 \mathrm{~s}$ exposures used to construct the SSDF mosaics, sources brighter than [3.6] $=[4.5] \sim 10$ Vega mag are saturated. Users of the SSDF catalogs are cautioned against uncritical usage of photometry for sources brighter than [3.6] $=[4.5]=$ 11.5 Vega mag. Objects that are truly as bright as this will be well-detected in any case by the two short-wavelength WISE bands.

\section{SUMMARY}

We have carried out an infrared survey of nearly $100 \mathrm{deg}^{2}$ with the warm IRAC aboard Spitzer for our Cycle 8 Spitzer Exploration program, the Spitzer South Pole Telescope Deep Field survey. With its combination of uniform depth in two infrared bands and wide-area coverage, this project provides a unique resource for extragalactic research. It benefits from numerous coextensive observations spanning $\mathrm{X}$-ray to radio wavelengths, in particular the deep imaging acquired by the SPT at $1.4,2$, and $3 \mathrm{~mm}$, and will therefore be of particular use for galaxy cluster science. The catalogs contain multiple photometric measurements for several million distinct IRAC sources down to the $5 \sigma$ survey limits of 7.0 and $9.4 \mu \mathrm{Jy}$ at 3.6 and $4.5 \mu \mathrm{m}$, respectively, and have been made publicly available to the astronomical community from the Spitzer Science Center.

This work is based on observations made with the Spitzer Space Telescope, which is operated by the Jet Propulsion Laboratory, California Institute of Technology under contract with the National Aeronautics and Space Administration (NASA). Support was provided by NASA through contract number 1439357 issued by JPL/Caltech. IRAF is distributed by the National Optical Astronomy Observatory, which is operated by the Association of Universities for Research in Astronomy (AURA) under cooperative agreement with the National Science Foundation. Lawrence Livermore National Laboratory is operated by Lawrence Livermore National Security, LLC, for the U.S. 
Table 7

SSDF IRAC Number Counts

\begin{tabular}{lcccc}
\hline \hline Mag & \multicolumn{2}{c}{$3.6 \mu \mathrm{m}$} & \multicolumn{2}{c}{$4.5 \mu \mathrm{m}$} \\
\cline { 2 - 3 } (Vega) & Counts & Unc. & Counts & Unc. \\
\hline 11.0 & $\ldots$ & $\ldots$ & 1.69 & 0.058 \\
11.5 & $\ldots$ & $\ldots$ & 1.79 & 0.052 \\
12.0 & 2.04 & 0.040 & 2.05 & 0.039 \\
12.5 & 2.20 & 0.033 & 2.20 & 0.033 \\
13.0 & 2.38 & 0.027 & 2.40 & 0.026 \\
13.5 & 2.54 & 0.023 & 2.57 & 0.022 \\
14.0 & 2.71 & 0.019 & 2.75 & 0.018 \\
14.5 & 2.90 & 0.015 & 2.97 & 0.014 \\
15.0 & 3.10 & 0.012 & 3.21 & 0.011 \\
15.5 & 3.38 & 0.009 & 3.49 & 0.008 \\
16.0 & 3.63 & 0.007 & 3.76 & 0.006 \\
16.5 & 3.87 & 0.005 & 4.00 & 0.004 \\
17.0 & 4.05 & 0.004 & 4.17 & 0.004 \\
17.5 & 4.20 & 0.003 & 4.32 & 0.003 \\
18.0 & 4.33 & 0.003 & 4.38 & 0.003 \\
18.5 & 4.49 & 0.002 & $\ldots$ & $\ldots$ \\
19.0 & 4.61 & 0.002 & $\ldots$ & $\ldots$ \\
\hline
\end{tabular}

Notes. Differential SSDF number counts measured in bins of width $0.5 \mathrm{mag}$ centered at the magnitudes given in the left-hand column. Counts are expressed in terms of $\log (N) \mathrm{mag}^{-1} \mathrm{deg}^{-2}$. All uncertainties are $1 \sigma$ and reflect Poisson counting statistics only; uncertainties arising from the incompleteness correction will dominate at faint levels.

Department of Energy, National Nuclear Security Administration under Contract DE-AC52-07NA27344. F.P. acknowledges support from grant 50 OR 1117 of the Deutches Zenturm für Luft- und Raumfahrt (DLR). We thank Dave Nair for his efforts in characterizing a preliminary reduction of the SSDF images. We also thank Richard G. Arendt, who kindly computed the Milky Way star count models shown in Figure 8.

Facility: Spitzer (IRAC)

\section{REFERENCES}

Arendt, R. G., Odegard, N., Weiland, J. L., et al. 1998, ApJ, 508, 74 Arnaud, M., Pointecouteau, E., \& Pratt, G. W. 2007, A\&A, 474, L37
Ashby, M. L. N., Stern, D., Brodwin, M., et al. 2009, ApJ, 701, 428

Austermann, J. E., Aird, K. A., Beall, J. A., et al. 2012, Proc. SPIE, 8452, $84521 \mathrm{E}$

Battaglia, N., Bond, J. R., Pfrommer, C., \& Sievers, J. L. 2013, ApJ, 777, 123

Bertin, E., \& Arnouts, S. 1996, A\&AS, 117, 393

Brodwin, M., Brown, M. J. I., Ashby, M. L. N., et al. 2006, ApJ, 651, 791

Brodwin, M., Ruel, J., Ade, P. A. R., et al. 2010, ApJ, 721, 90

Carlstrom, J. E., Ade, P. A. R., Aird, K. A., et al. 2011, PASP, 123, 568

Cohen, M. 1993, AJ, 105, 1860

Cohen, M. 1994, AJ, 107, 582

Cohen, M. 1995, ApJ, 444, 874

Desai, S., Armstrong, R., Mohr, J. J., et al. 2012, ApJ, 757, 83

Eisenhardt, P. R. M., Brodwin, M., Gonzalez, A. H., et al. 2008, ApJ, 684,905

Eisenhardt, P. R. M., Stern, D., Brodwin, M., et al. 2004, ApJS, 154, 48

Fazio, G. G., Ashby, M. L. N., Barmby, P., et al. 2004a, ApJS, 154, 39

Fazio, G. G., Hora, J. L., Allen, L. E., et al. 2004b, ApJS, 154, 10

Finkbeiner, D. P., Davis, M., \& Schlegel, D. J. 1999, ApJ, 524, 867

Giodini, S., Pierini, D., Finoguenov, A., et al. 2009, ApJ, 703, 982

Gonzalez, A. H., Zaritsky, D., \& Zabludoff, A. I. 2007, ApJ, 666, 147

Griffin, M. J., Abergel, A., Abreu, A., et al. 2010, A\&A, 518, L3

Hinshaw, G., Larson, D., Komatsu, E., et al. 2013, ApJS, 208, 19

Holder, G. P., Viero, M. P., Zahn, O., et al. 2013, ApJL, 771, L16

Kravtsov, A., Gonzalez, A., Vikhlinin, A., et al. 2009, astro2010: The Astronomy and Astrophysics Decadal Survey, 164

Lin, L., Dickinson, M., Jian, H.-Y., et al. 2012, ApJ, 756, 71

Lonsdale, C. J., Smith, H. E., Rowan-Robinson, M., et al. 2003, PASP, 115, 897

Mauduit, J. C., Lacy, M., Farrah, D., et al. 2012, PASP, 124, 714

Muzzin, A., Wilson, G., Yee, H. K. C., et al. 2009, ApJ, 698, 1934

Muzzin, A., Wilson, G., Yee, H. K. C., et al. 2012, ApJ, 746, 188

Papovich, C., Momcheva, I., Willmer, C. N. A., et al. 2010, ApJ, 716, 1503

Pierre, M., Pacaud, F., Juin, J. B., et al. 2011, MNRAS, 414, 1732

Planck Collaboration, Ade, P. A. R., Aghanim, N., et al. 2013, arXiv:1303.5076

Planelles, S., Borgani, S., Dolag, K., et al. 2013, MNRAS, 431, 1487

Quijada, M. A., Marx, C. T., Arendt, R. G., \& Moseley, S. H. 2004, Proc. SPIE, 5487, 244

Reichardt, C. L., Stalder, B., Bleem, L. E., et al. 2013, ApJ, 763, 127

Schuster, M. T., Marengo, M., \& Patten, B. M. 2006, Proc. SPIE, 6270, 65

Skrutskie, M. F., Cutri, R. M., Stiening, R., et al. 2006, AJ, 131, 1163

Stanford, S. A., Brodwin, M., Gonzalez, A. H., et al. 2012, ApJ, 753, 164

Story, K. T., Reichardt, C. L., Hou, Z., et al. 2012, arXiv:1210.7231S

Sun, M., Voit, G. M., Donahue, M., et al. 2009, ApJ, 693, 1142

Sunyaev, R. A., \& Zel'dovich, Y. B. 1972, CoASP, 4, 173

Vieira, J. D., Marrone, D. P., Chapman, S. C., et al. 2013, Natur, 495, 344

Vikhlinin, A., Kravtsov, A., Forman, W., et al. 2006, ApJ, 640, 691

Wainscoat, R. J., Cohen, M., Volk, K., et al. 1992, ApJS, 83, 111

Wright, E. L., Eisenhardt, P. R. M., Mainzer, A. K., et al. 2010, AJ, 140, 1868 